\title{
Effect of Microscopic Third Ventriculostomy (Lamina Terminalis Fenestration) on Shunt-needed Hydrocephalus in Patients with Aneurysmal Subarachnoid Hemorrhage
}

\author{
Alireza Tabibkhooei', Maziar Azar', Morteza Taheri', \\ Hossein Ghalaenovi', Arash Fattahi ${ }^{2}$, Hamed Kheradmand ${ }^{1}$ \\ ${ }^{1}$ Department of Neurosurgery, Iran University of Medical Sciences, Rasool \\ Akram Hospital, Tehran, Iran; \\ ${ }^{2}$ Department of Neurosurgery, Iran University of Medical Sciences, 7Tir Hospital, \\ Tehran, Iran
}

Received July 17, 2020; Accepted February 5, 2021.

Key words: Aneurysmal subarachnoid hemorrhage - Acute hydrocephalus - Shuntneeded hydrocephalus - Lamina terminalis fenestration - Ventriculostomy

\begin{abstract}
There are reports that in patients with aSAH (aneurysmal subarachnoid hemorrhage), LTF (lamina terminalis fenestration) reduces the rate of shunt-needed hydrocephalus via facilitation of CSF (cerebrospinal fluid) dynamic, diminished leptomeningeal inflammation, and decreased subarachnoid fibrosis. Regarding the conflicting results, this study was conducted to evaluate the effects of LTF on decreased shunt-needed hydrocephalus in patients with aSAH. A cross-sectional retrospective study was carried out to survey all patients with confirmed aSAH operated from March 2011 to September 2016 in an academic vascular center (Rasool Akram Hospital in Tehran, Iran). Of a total of 151 patients, 72 patients were male and 79 were female. The mean age of the participants was 51 years. A transiently CSF diversion (EVD - external ventricular drainage) was performed (the acute hydrocephalus rate) on 21 patients (13.9\%). In 36 patients (23.8\%), aneurysm occlusion with LTF and in 115 patients (76.2\%) only aneurysm occlusion surgery was performed. In hydrocephalus follow-up after surgery, 13 (12\%) patients needed shunt insertion (the rate of shunt-needed hydrocephalus). The statistical analysis demonstrated no significant relation between LTF and shunt-needed hydrocephalus. Confirmation of the hypothesis that LTF may decrease the rate of shunt-needed hydrocephalus can significantly decrease morbidity, mortality, and
\end{abstract}

Mailing Address: Dr. Morteza Taheri, Department of Neurosurgery, Iran University of Medical Sciences, 7Tir Hospital, Tehran, Iran; Phone: +989 120194 908; Fax: 021552179 01; e-mail: drtaheri38@yahoo.com 
treatment costs of shunting (that is a simple, but a potentially dangerous procedure). So, it is advised to plan and perform an RCT (randomized controlled trial) that can remove the confounding factors, match the groups, and illustrate the exact effect of LTF on shunt-needed hydrocephalus.

\section{Introduction}

Subarachnoid hemorrhage (SAH) is defined as the presence of blood within the cerebral cisterns and comprises about five percent of all cerebrovascular strokes. The most common cause of spontaneous SAH is the rupture of the intracranial aneurysms, aneurysmal SAH (aSAH) (Winn, 2016). The prevalence of this stroke is about 7-9 per 100,000 person-year (Woernle et al., 2013; Winn, 2016). The prevalence increases with higher age, and it is more frequent in women than men in the sixth decade of life. Despite the persisted prevalence, the mortality rate has decreased, and the estimated case fatality rate is $23-67 \%$, with a $5 \%$ decrease per year (Winn, 2016).

One of the most important complications following aSAH in patients survived from the acute phase of the disease is hydrocephalus, with a rate of $6-67 \%$. Increased age, being a female, high Hunt and Hess $(\mathrm{H} \& H)$ scale on admission, thick $\mathrm{SAH}$ on initial brain computed tomography (CT) scan, intraventricular hemorrhage $(\mathrm{IVH})$, hydrocephalus in initial brain CT scan, posterior circulation aneurysm, the presence of clinical vasospasm, and endovascular treatment are factors related to the shunt-depended hydrocephalus (Nam et al., 2010; Woernle et al., 2013; Bae et al., 2014).

According to the studies, most important mechanisms that result in hydrocephalus include disturbance in cerebrospinal fluid (CSF) dynamic, obstructive mechanisms due to the presence of blood, disruption in the absorptive mechanisms at the level of arachnoid granulations, and hemorrhage induced inflammation (Komotar et al., 2002; Demirgil et al., 2003; Dehdashti et al., 2004; Adams et al., 2016).

It has been demonstrated that 6 to $45 \%$ of patients with acute hydrocephalus require shunt insertion, ultimately (Tapaninaho et al., 1993; Zaidi et al., 2015). Although shunt insertion is a benign procedure, it can be accompanied by serious complications such as shunt failure, shunt infection, and repetitive admission (Tapaninaho et al., 1993; Zaidi et al., 2015). The rate of shunt failure and other complications have been reported up to $40 \%$ for the first year and up to $85 \%$ for the following 10 years (Komotar et al., 2002).

In recent years, reports show that lamina terminalis fenestration (LTF) decreases the rate of shunt-needed hydrocephalus via the facilitation of CSF dynamic, diminished leptomeningeal inflammation, and decreased subarachnoid fibrosis (Komotar et al., 2002). Regarding the conflicting results of the effect of LTF on shunt-needed hydrocephalus, this study was conducted to evaluate the effects of LTF on decreased shunt-needed hydrocephalus in patients with aSAH in an academic center. 


\section{Material and Methods}

A cross-sectional retrospective study was performed to survey all patients with confirmed aSAH operated from March 2011 to September 2016 in an academic vascular center (Rasool Akram Hospital in Tehran, Iran). The intended data were collected via the admission hospital files and hospital imaging archives.

All patients with a diagnosis of SAH were included in the study, and the patients with SAH without confirmed aSAH, the patients with confirmed aSAH who did not undergo operation for any reason, and the patients with deficient data were excluded from the study.

Four-vessel brain digital subtraction angiography (DSA) was performed for all patients, and an aneurysm as a reason for SAH was confirmed.

According to the routine management of patients with SAH in our center, brain angiography and surgery were performed very soon. Angiography in the same day or the day after admission and surgery in the same day or the day after performing the angiography generally depend on the clinical and medical condition of the patients.

Considering treatment, all the patients underwent craniotomy and microsurgical clipping of the aneurysm. But, LTF (third ventriculostomy) was performed for some patients but not all.

LTF or third ventriculostomy was performed after the aneurysm clipping via the microscopic opening of the anterior wall of the third ventricle located posterior to the optic chiasma.

To define the clinical status of the patients, clinical scales including the Glasgow Coma Scale (GCS), World Federation of Neurosurgical Societies (WFNS), and H\&H scales were used, and for grading SAH on CT scan, Fisher grade was used.

The sample size included all patients with aSAH in the aforementioned period. After collecting the data and required variables, the data were analysed using SPSS software by a statistic specialist.

Using the statistical formula including chi-square, fissure, independent $t$-test, and logistic regression, the data analysed. The significant value for this study was 0.05 .

\section{Ethical considerations}

Regarding the retrospective nature of this study, we had no intervention or effect on the patients and patients' outcomes. Moreover, the institutional ethics committee of the Iran University of Medical Sciences approved the study. The informed consent was obtained from all participants.

\section{Results}

Considering the inclusion criteria, a total of 320 patients enrolled. After applying the exclusion criteria, 151 patients who underwent aneurysmal occlusion surgery (surgical clipping) were surveyed (Figure 1). 


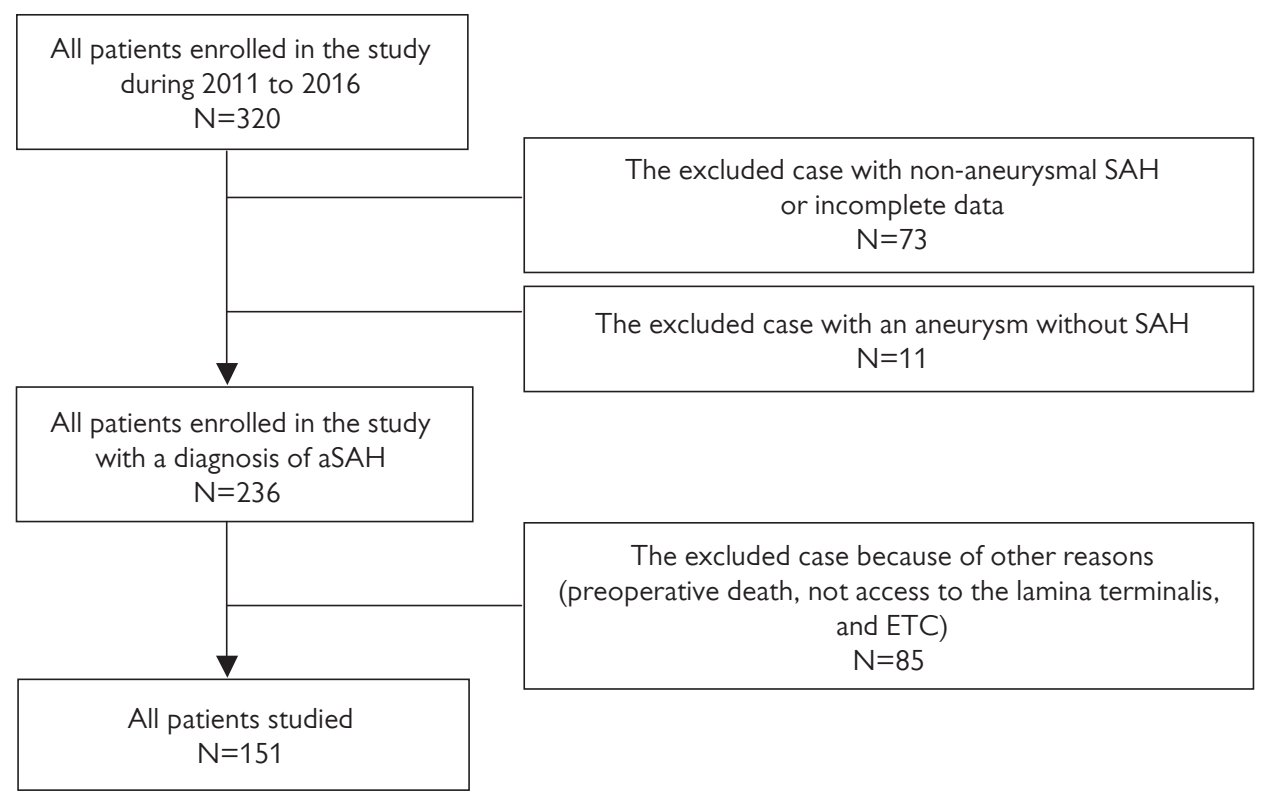

Figure 1 - The consort flow chart of the patients.

The mean interval between admission and surgery was 3.9 days within a range of 1 to 18 days. Of a total of 151 patients, 72 patients (47.7\%) were males and 79 (52.3\%) were females. The mean age was 51 . In 21 patients (13.9\%), a transiently CSF diversion (EVD - external ventricular drainage) was performed due to acute

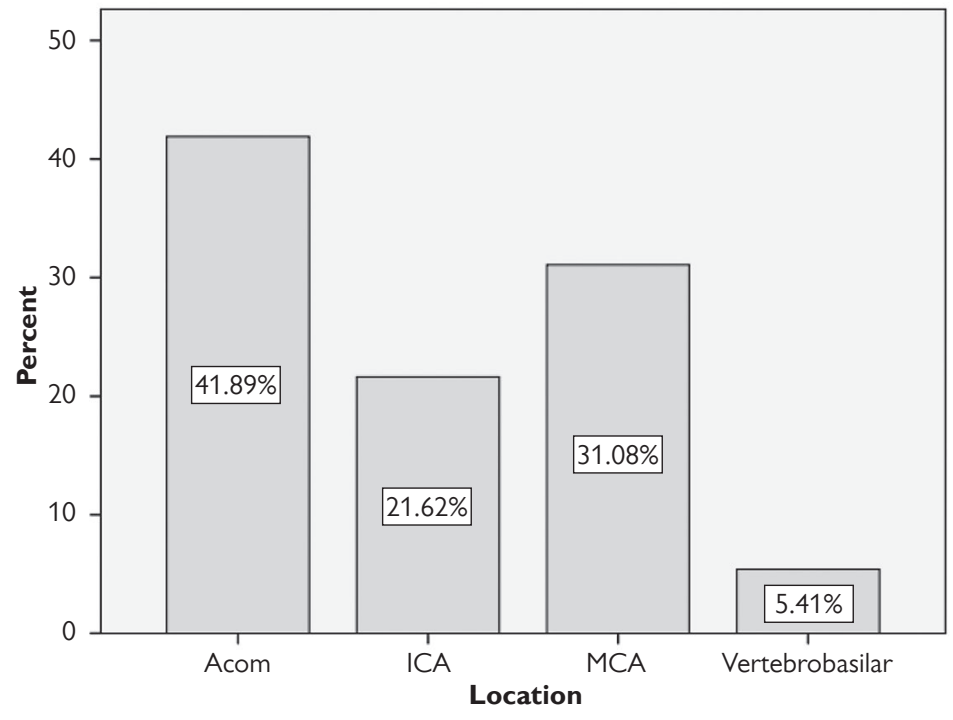

Figure 2 - The location of an aneurysm. 
Table 1 - Comparison of some features of two groups: LTF and not LTF

\begin{tabular}{|c|c|c|c|c|}
\hline Parameters & & LTF $(n=36)$ & No LTF $(n=115)$ & P-value \\
\hline Age, mean $\pm S D$ & & $54.42 \pm 11.04$ & $49.97 \pm 13.10$ & $>0.05$ \\
\hline \multirow{2}{*}{ Sex } & male & 17 & 55 & \multirow{2}{*}{$>0.05$} \\
\hline & female & 19 & 59 & \\
\hline $\mathrm{GCS}$, mean $\pm \mathrm{SC}$ & & 12.69 & 13.58 & $>0.05$ \\
\hline \multirow{5}{*}{$\begin{array}{l}\text { Hunt and } \\
\text { Hess grade }\end{array}$} & 1 & 4 & 18 & \multirow{5}{*}{$>0.05$} \\
\hline & 2 & 11 & 42 & \\
\hline & 3 & 12 & 40 & \\
\hline & 4 & 6 & 14 & \\
\hline & 5 & 3 & 1 & \\
\hline \multirow{5}{*}{$\begin{array}{l}\text { WFNS } \\
\text { grade }\end{array}$} & 1 & 13 & 54 & \multirow{5}{*}{$<0.05$} \\
\hline & 2 & 12 & 34 & \\
\hline & 3 & 3 & 8 & \\
\hline & 4 & 4 & 18 & \\
\hline & 5 & 4 & 1 & \\
\hline \multirow{4}{*}{ Fisher grade } & 1 & 1 & 6 & \multirow{4}{*}{$>0.05$} \\
\hline & 2 & 9 & 32 & \\
\hline & 3 & 14 & 38 & \\
\hline & 4 & 12 & 39 & \\
\hline \multirow{2}{*}{$\mathrm{IVH}$} & yes & 10 & 19 & \multirow{2}{*}{$>0.05$} \\
\hline & no & 26 & 95 & \\
\hline \multirow{2}{*}{$\begin{array}{l}\text { Hydrocephalus } \\
\text { on admission } \\
\text { (EVD insertion) }\end{array}$} & yes & 7 & 14 & \multirow{2}{*}{$>0.05$} \\
\hline & no & 29 & 101 & \\
\hline
\end{tabular}

LTF - lamina terminalis fenestration; SD - standard deviation; GCS - Glasgow Coma Scale; WFNS - World Federation of Neurosurgical Societies; IVH - intraventricular hemorrhage; EVD - external ventricular drainage

hydrocephalus (the rate of acute hydrocephalus). Anterior communicating artery (ACOM) was the most common location of the aneurysm (Figure 2). In 36 patients (23.8\%), aneurysm occlusion surgery with LTF (LTF group) and in 115 patients (76.2\%) only aneurysm occlusion surgery (not LTF group) were performed. Totally, 105 patients (69.5\%) were discharged and 46 patients (30.5\%) died. In follow-up for hydrocephalus after surgery, 43 patients died, and 13 (12\%) patients needed shunt insertion (the rate of shunt-needed hydrocephalus).

Table 1 shows the most important baseline features of the patients in two groups. Comparing the two groups revealed no significant difference.

Comparing the effect LTF on shunting demonstrated that although the rate of shunt-needed hydrocephalus differed between the two groups of LTF and not LTF, this difference was not statistically significant. Moreover, the analysis showed no positive effects of LTF on other variables (Table 2). 
Table 2 - Statistical relation between LTF and some study variables

\begin{tabular}{|c|c|c|c|c|c|}
\hline \multirow{2}{*}{ Variable } & & \multicolumn{2}{|c|}{ LTF } & \multirow{2}{*}{ Total } & \multirow{2}{*}{ P-value } \\
\hline & & yes & no & & \\
\hline \multirow{2}{*}{ Shunting } & yes & $15.4 \%$ & $11.0 \%$ & $12.0 \%$ & \multirow{2}{*}{$>0.05$} \\
\hline & no & $84.6 \%$ & $89.0 \%$ & $88.0 \%$ & \\
\hline \multirow{2}{*}{ Outcome } & discharge & $67.0 \%$ & $70.0 \%$ & $69.5 \%$ & \multirow{2}{*}{$>0.05$} \\
\hline & death & $33.0 \%$ & $30.0 \%$ & $30.5 \%$ & \\
\hline \multicolumn{2}{|c|}{ Hospitalization (days) } & 27.06 & 23.38 & & $>0.05$ \\
\hline
\end{tabular}

LTF - lamina terminalis fenestration

\section{Discussion}

Hydrocephalus is one of the most important complications in patients who survive following the acute phase of aSAH. Some factors are related to the shunt-needed hydrocephalus including increased age, being a female, high $\mathrm{H} \& \mathrm{H}$ grade on admission, thick SAH on brain CT scan, IVH, hydrocephalus on first brain CT scan, posterior circulation aneurysm, clinical vasospasm, and endovascular treatment (Nam et al., 2010; Woernle et al., 2013; Bae et al., 2014).

The hydrocephalus after SAH occurred in up to $67 \%$ of the patients. It is divided into three subgroups according to the time of occurrence: acute (up to day 3 ), subacute (day 4 to 13), and chronic (more than 14 days) (Dorai et al., 2003; de Oliveira et al., 2007; Yang et al., 2013). In the acute phase, some of these patients require the emergency external ventricular drainage to decrease the ICP (intracranial pressure). Nevertheless, this acute condition can be spontaneously resolved, or in some patients, the CSF dynamic interfering mechanisms result in continued raised ICP that require permanent CSF diversion intervention (Adams et al., 2016).

CSF dynamic disruption is one of the most important reasons for persistent hydrocephalus. Some studies reported that disruption in CSF dynamic, obstructive mechanism due to the presence of blood, disruption in the absorptive mechanisms at the arachnoid granules levels, and hemorrhage induced inflammation are the most important mechanisms that result in hydrocephalus (Adams et al., 2016). SAH results in permanent hydrocephalus in about $20 \%$ of patients due to leptomeningeal fibrosis and arachnoidal granules fibrosis and resultantly decreased CSF absorption (Komotar et al., 2002).

It is noted that 6 to $45 \%$ of acute hydrocephalus require shunting. Although the shunt insertion accounts for a benign process, it can be associated with procedurerelated complications such as shunt failure, infection, repetitive admission, and etc. (Tapaninaho et al., 1993; Zaidi et al., 2015). Therefore, the risk of shunt failure is up to $40 \%$ in the first year, and up to $85 \%$ in the following 10 years (Komotar et al., 2002). 


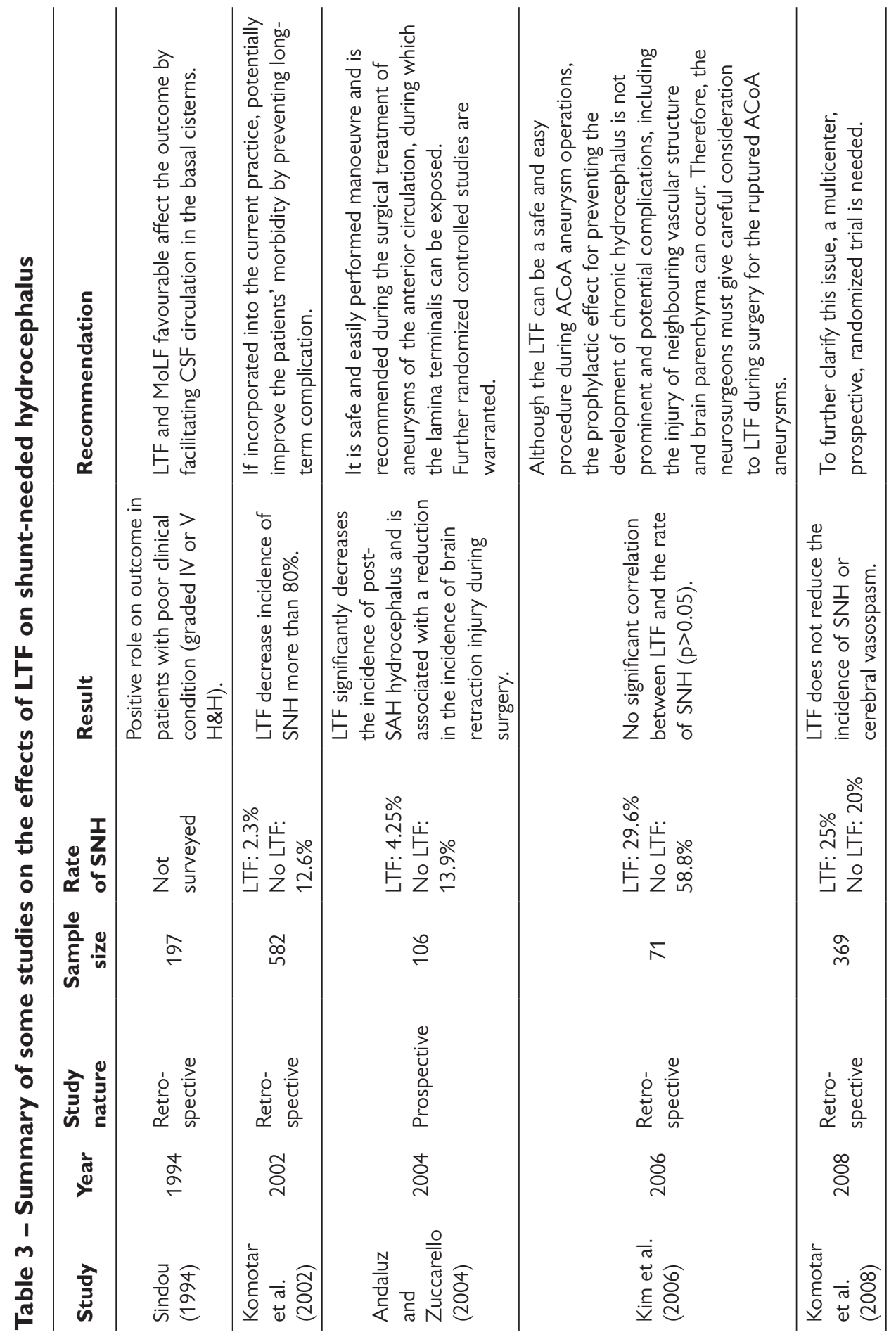




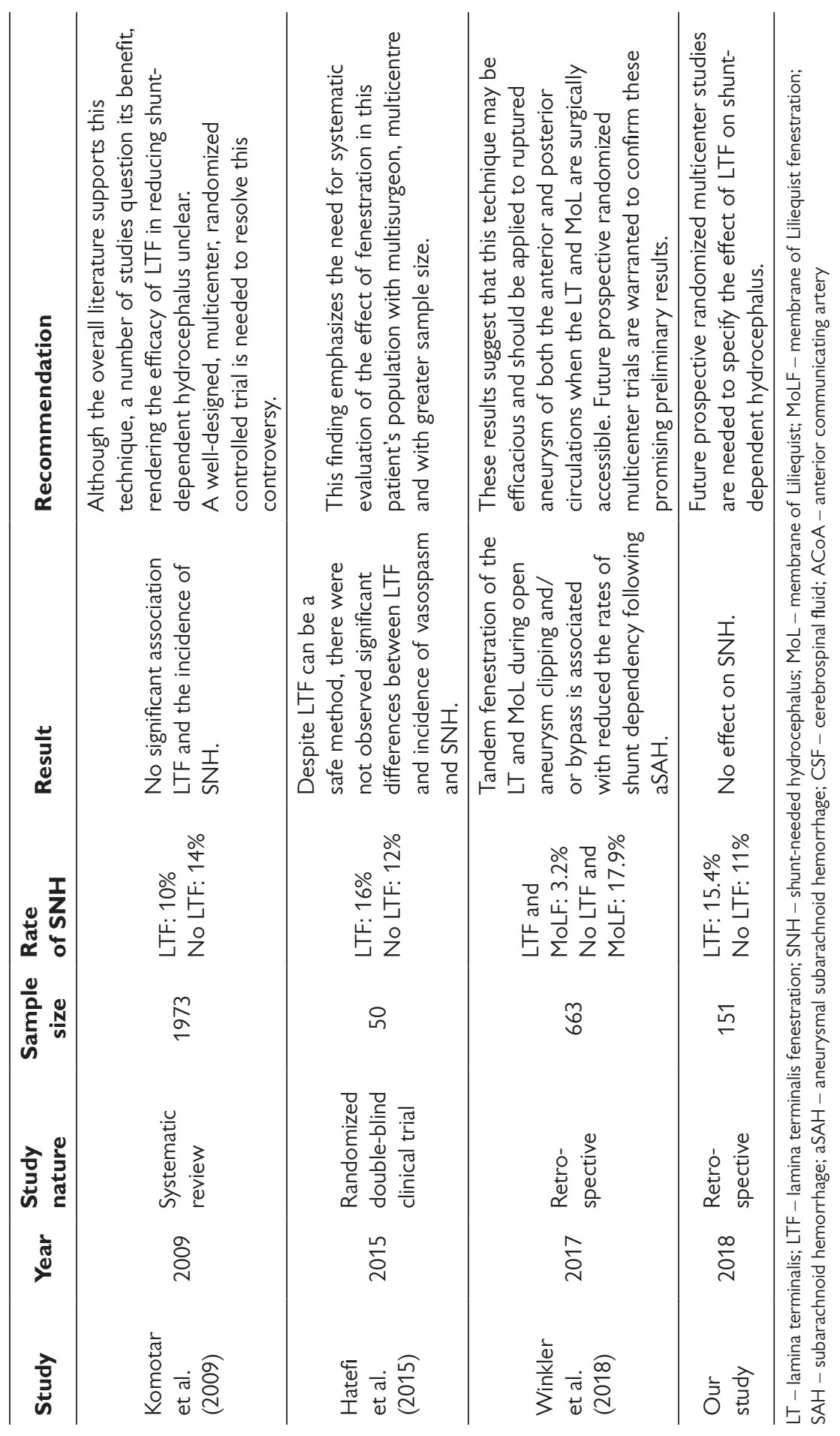


Sugawara et al. (2016) reported that $33.8 \%$ of their patients required shunting for shunt-needed hydrocephalus (10.7\% hydrocephalus with high pressure and $23 \%$ hydrocephalus with normal pressure).

Adams et al. (2016) surveyed 1,553 patients who survived 14 days after SAH. The rate of permanent CSF diversion was $17.7 \%$. The mean follow-up was 8 years. Patients who required shunting had higher age, higher $\mathrm{H} \& \mathrm{H}$, and fisher grade on admission, needed more EVD insertion and endovascular treatment, and meningitis and IVH occurred more in these patients. The mean time to shunt insertion was 33 days. The patients who underwent shunting within the first month were younger, female, and hydrocephalus on the first brain CT scan compared to the patients who underwent shunt after 30 days. Multivariate analysis demonstrated that the age at admission, size of an aneurysm, hydrocephalus on the first brain CT scan, and meningitis were the independent risk factors for shunt insertion (Adams et al., 2016).

Komotar et al. published their paper in 2008. The rates of shunt insertion, shunt conversion, and clinical vasospasm were, respectively, 25,50 , and $23 \%$ in patients who underwent LTF and 20, 27, and $27 \%$ in patients without LTF. They concluded that LTF probably did not have any effect on decreasing the rate of shunt insertion and vasospasm (Komotar et al., 2008).

Andaluz and Zuccarello (2004) demonstrated that LTF results in a decrease in the rate of shunting and vasospasm and improves patients after aSAH.

Hatefi et al. (2015) demonstrated that the demographic features, H\&H and fissure grades, the rate of shunt insertion, and clinical vasospasm are the same in groups with LTF and without LTF.

The rate of acute hydrocephalus needed for surgical intervention for diminishing ICP was $13.9 \%$. A wide range has been reported for this rate, from $15 \%$ in the study of Komotar et al. (2008) to 63.6\% in the study of Czorlich et al. (2015). Comparing the results and other studies demonstrated the lower rate of acute hydrocephalus in the present study. Although a part of this difference can be explained with a different and relative definition of hydrocephalus and difference in planning for surgical treatment in various centers, planning a suitable clinical trial for surveying the definite rate of acute hydrocephalus, exhibiting differences, and illustrating the reasons for its low rate in the patients are required.

The rate of chronic hydrocephalus in our study was $12 \%$. This rate was within a wide range from $8.4 \%$ in the study of Andaluz and Zuccarello (2004) to $37 \%$ in the study of Yoshioka et al. (2000), too. Comparing these results and other studies demonstrated the lower rate of chronic hydrocephalus in the present study similar to acute hydrocephalus.

The present study revealed no positive effects of LTF on decreasing the rate of shunt-needed hydrocephalus. Probably, the low rate of acute hydrocephalus has an effect on the results. The review of the literature demonstrated paradoxical results; some reported positive effects and some reported no effects. 
Although a prospective study (Andaluz and Zuccarello, 2004) demonstrated that the LTF significantly decreases the incidence of post-SAH hydrocephalus, another study (Komotar et al., 2009) reported no positive effect in a systematic review after analysis of 1,973 patients. The only randomized double-blind clinical trial performed demonstrated no positive effect. Table 3 compares the most important studies performed in this setting.

\section{Conclusion}

In summary, the rate of acute hydrocephalus and chronic hydrocephalus was 13.9 and $12 \%$, respectively. LTF demonstrated no effect on decreasing the rate of chronic hydrocephalus.

Confirming the hypothesis that LTF decreases shunt-needed hydrocephalus significantly reduces morbidity, mortality, and treatment costs of shunting (a simple but potentially dangerous procedure). It is advised to plan and perform a randomized double-blind clinical trial with larger sample size to remove the confounding factors and homogenize the groups to illustrate the exact effect of LTF on shunt-needed hydrocephalus.

\section{References}

Adams, H., Ban, V. S., Leinonen, V., Aoun, S. G., Huttunen, J., Saavalainen, T., Lindgren, A., Frosen, J., Fraunberg, M., Koivisto, T., Hernesniemi, J., Welch, B. G., Jaaskelainen, J. E., Huttunen, T. J. (2016) Risk of shunting after aneurysmal subarachnoid hemorrhage: A collaborative study and initiation of a consortium. Stroke 47(10), 2488-2496.

Andaluz, N., Zuccarello, M. (2004) Fenestration of the lamina terminalis as a valuable adjunct in aneurysm surgery. Neurosurgery 55(5), 1050-1059.

Bae, I. S., Yi, H. J., Choi, K. S., Chun, H. J. (2014) Comparison of incidence and risk factors for shuntdependent hydrocephalus in aneurysmal subarachnoid hemorrhage patients. J. Cerebrovasc. Endovasc. Neurosurg. 16(2), 78-84.

Czorlich, P., Ricklefs, F., Reitz, M., Vettorazzi, E., Abboud, T., Regelsberger, J., Westphal, M., Schmidt, N. O. (2015) Impact of intraventricular hemorrhage measured by Graeb and LeRoux score on case fatality risk and chronic hydrocephalus in aneurysmal subarachnoid hemorrhage. Acta Neurochir. (Wien) 157(3), 409-415.

de Oliveira, J. G., Beck, J., Setzer, M., Gerlach, R., Vatter, H., Seifert, V., Raabe, A. (2007) Risk of shuntdependent hydrocephalus after occlusion of ruptured intracranial aneurysms by surgical clipping or endovascular coiling: a single-institution series and meta-analysis. Neurosurgery 61(5), 924-933; discussion 933-934.

Dehdashti, A. R., Rilliet, B., Rufenacht, D. A., De Tribolet, N. (2004) Shunt-dependent hydrocephalus after rupture of intracranial aneurysms: A prospective study of the influence of treatment modality.

J. Neurosurg. 101(3), 402-407.

Demirgil, B. T., Tugcu, B., Postalci, L., Guclu, G., Dalgic, A., Oral, Z. (2003) Factors leading to hydrocephalus after aneurysmal subarachnoid hemorrhage. Minim. Invasive Neurosurg. 46(6), 344-348.

Dorai, Z., Hynan, L. S., Kopitnik, T. A., Samson, D. (2003) Factors related to hydrocephalus after aneurysmal subarachnoid hemorrhage. Neurosurgery 52(4), 763-769.

Hatefi, M., Azhary, S., Naebaghaee, H., Mohamadi, H. R., Jaafarpour, M. (2015) The effect of fenestration 
of lamina terminalis on the vasospasm and shunt-dependent hydrocephalus in patients following subarachnoid haemorrhage. J. Clin. Diagn. Res. 9(7), PC15-PC18.

Kim, J. M., Jeon, J. Y., Kim, J. H., Cheong, J. H., Bak, K. H., Kim, C. H., Yi, H. J., Kim, K. M. (2006) Influence of lamina terminalis fenestration on the occurrence of the shunt-dependent hydrocephalus in anterior communicating artery aneurysmal subarachnoid hemorrhage. J. Korean Med. Sci. 21(1), 113-118.

Komotar, R. J., Olivi, A., Rigamonti, D., Tamargo, R. J. (2002) Microsurgical fenestration of the lamina terminalis reduces the incidence of shunt-dependent hydrocephalus after aneurysmal subarachnoid hemorrhage. Neurosurgery 51(6), 1403-1412; discussion 1412-1413.

Komotar, R. J., Hahn, D. K., Kim, G. H., Khandji, J., Mocco, J., Mayer, S. A., Connolly, E. S. Jr. (2008) The impact of microsurgical fenestration of the lamina terminalis on shunt-dependent hydrocephalus and vasospasm after aneurysmal subarachnoid hemorrhage. Neurosurgery 62(1), 123-132; discussion 132-134

Komotar, R. J., Hahn, D. K., Kim, G. H., Starke, R. M., Garrett, M. C., Merkow, M. B., Otten, M. L., Sciacca, R. R., Connolly, E. S. Jr. (2009) Efficacy of lamina terminalis fenestration in reducing shunt-dependent hydrocephalus following aneurysmal subarachnoid hemorrhage: a systematic review. Clinical article. J. Neurosurg. 111(1), 147-154.

Nam, K. H., Hamm, I. S., Kang, D. H., Park, J., Kim, Y. S. (2010) Risk of shunt dependent hydrocephalus after treatment of ruptured intracranial aneurysms: Surgical clipping versus endovascular coiling according to fisher grading system. J. Korean Neurosurg. Soc. 48(4), 313-318.

Sindou, M. (1994) Favourable influence of opening the lamina terminalis and Lilliequist's membrane on the outcome of ruptured intracranial aneurysms. A study of 197 consecutive cases. Acta Neurochir. (Wien) 127(1-2), 15-16.

Sugawara, T., Maehara, T., Nariai, T., Aoyagi, M., Ohno, K. (2016) Independent predictors of shunt-dependent normal pressure hydrocephalus after aneurysmal subarachnoid hemorrhage. J. Neurosurg. Sci. 60(2), 154-158.

Tapaninaho, A., Hernesniemi, J., Vapalahti, M., Niskanen, M., Kari, A., Luukkonen, M., Puranen, M. (1993) Shunt-dependent hydrocephalus after subarachnoid haemorrhage and aneurysm surgery: Timing of surgery is not a risk factor. Acta Neurochir. (Wien) 123(3-4), 118-124.

Winkler, E. A., Burkhardt, J. K., Rutledge, W. C., Rick, J. W., Partow, C. P., Yue, J. K., Birk, H., Bach, A. M., Raygor, K. P., Lawton, M. L. (2018) Reduction of shunt dependency rates following aneurysmal subarachnoid hemorrhage by tandem fenestration of the lamina terminalis and membrane of Liliequist during microsurgical aneurysm repair. J. Neurosurg. 129(5), 1166-1172.

Winn, H. R. (2016) Youmans and Winn Neurological Surgery, $7^{\text {th }}$ Ed. Elsevier, Philadelphia.

Woernle, C. M., Winkler, K. M. L., Burkhardt, J. K., Haile, S. R., Bellut, D., Neidert, M. C., Bozinov, O., Krayenbühl, N., Bernays, R. L. (2013) Hydrocephalus in 389 patients with aneurysm-associated subarachnoid hemorrhage. J. Clin. Neurosci. 20(6), 824-826.

Yang, T. C., Chang, C. H., Liu, Y. T., Chen, Y. L., Tu, P. H., Chen, H. C. (2013) Predictors of shunt-dependent chronic hydrocephalus after aneurysmal subarachnoid haemorrhage. Eur. Neurol. 69(5), 296-303.

Yoshioka, H., Inagawa, T., Tokuda, Y., Inokuchi, F. (2000) Chronic hydrocephalus in elderly patients following subarachnoid hemorrhage. Surg. Neurol. 53(2), 119-124; discussion 124-125.

Zaidi, H. A., Montoure, A., Elhadi, A., Nakaji, P., McDougall, C. G., Albuquerque, F. C., Spetzler, R. F., Zabramski, J. M. (2015) Long-term functional outcomes and predictors of shunt-dependent hydrocephalus after treatment of ruptured intracranial aneurysms in the BRAT trial: revisiting the clip vs coil debate. Neurosurgery 76(5), 608-613; discussion 613-614; quiz 164. 